\title{
Endogenous dissemination of genital Herpesvirus hominis type 2 to the eye
}

\author{
KAREN D. SUMERS, JOEL SUGAR, AND ROBERT LEVINE \\ From the Department of Ophthalmology, University of Illinois Eye and Ear Infirmary, \\ Chicago, Illinois 60612, USA
}

SUMMARY A 33-year-old man developed Herpesvirus hominis type 2 (HVH-2) eye disease following a herpetic lesion of the penis. The sequence of ocular involvement suggested that the virus had been transmitted endogenously from the genital lesion: granulomatous iritis was followed by interstitial keratitis and then by dendritic keratitis. Recurrent bacterial ulcers ultimately required a conjunctival flap. The course of this man's ocular disease as well as those of other reported cases of HVH-2 adult eye infections appeared to be more severe and prolonged than that of the average patient with ocular herpes type 1 infection.

Although most herpetic ocular infections in adults are caused by Herpesvirus hominis type 1 (HVH-1), and the majority of genital disease is secondary to HVH-2, exceptions to these rules occur. ${ }^{1-3}$ Fourteen previous cases have been reported of ocular HVH-2 infection in adults. ${ }^{14-7}$ We report an additional case that provides strong evidence for endogenous transmission of genital HVH-2 to the eye.

\section{Case report}

On 11 March 1977 a 33-year-old white man developed a blister on the shaft of his penis 1 week after sexual activity with a new partner. A urologist who examined him 4 days later diagnosed genital herpes simplex. The genital lesion healed during the next few days, but on 22 March 1977 the patient consulted a general physician for redness and discomfort in his left eye. He was treated with corticosteroid eye drops and was referred to an ophthalmologist 2 days later. The right eye was normal by slit-lamp examination. The left eye showed a dense cellular anterior chamber reaction. The cornea had numerous and large keratic precipitates, but there was no evidence of epithelial or stromal involvement. The intraocular pressure was $60 \mathrm{mmHg}$. The patient was started on a regimen of topical corticosteroids, cycloplegics, and acetazolamide. Three days later, on 29 March 1977, his

Correspondence to Dr Joel Sugar, University of Illinois Eye and Ear Infirmary, Department of Ophthalmology, 1855 West Taylor Street, Chicago, Illinois 60612, USA. intraocular pressure had decreased to $18 \mathrm{mmHg}$, but the anterior chamber reaction persisted. The patient received a subconjunctival corticosteroid injection, which produced some improvement. When next seen, on 9 April 1977, he was given a second subconjunctival injection of corticosteroids for an exacerbation of the anterior chamber inflammation. On 12 April 1977 interstitial keratitis and stromal infiltration were first noted in the superonasal quadrant of the cornea, but no epithelial disease was seen.

On 20 April 1977 visual acuity was 6/24 (20/80). The interstitial keratitis and a mild anterior chamber reaction persisted despite cycloplegia, topical corticosteroids, and idoxuridine (IDU). A week later bullous keratopathy and epithelial stippling were noted. Vidarabine (Vira-A) was substituted for IDU. On 6 May 1977 a flare-up of the iritis necessitated additional subconjunctival corticosteroids. The inflammation of the anterior chamber quieted somewhat, but the area of interstitial keratitis seemed to be enlarging. It was at this time that we first saw the patient.

Laboratory studies performed by the referring physician included a fluorescent treponemal antibody absorption test, erythrocyte sedimentation rate, and herpes titres at 3 and 7 weeks after the onset of ocular symptoms. All were reported as negative. A repeat herpes simplex complement fixation titre in our laboratory was $1: 64$.

The patient's past medical history was remarkable only for a long history of mild psoriasis, childhood rheumatic fever, and admission to hospital for 
fever of unknown origin in his early $20 \mathrm{~s}$. He had had bilateral 'pink eye' 2 years earlier. The patient denied ever having had labial herpes.

Our initial examination on 16 May 1977 revealed a visual acuity of $6 / 6(20 / 20)$ in the right eye and counting fingers at 2 feet $(60 \mathrm{~cm})$ in the left eye. The right eye was normal on external, slit-lamp, and ophthalmoscopic examinations. The left eye showed a slightly injected conjunctiva with steroid deposition inferiorly. The corneal sensitivity was normal. Slit-lamp examination showed diffuse bullous keratopathy with marked stromal thickening, haze, and a moderate number of folds in Descemet's membrane. There were no epithelial defects. The central corneal thickness was $0.88 \mathrm{~mm}$. A few keratic precipitates appeared to be inactive. The anterior chamber was deep and clear. The iris was not atrophic. The lens was clear. Intraocular pressûre was $23 \mathrm{mmHg}$ by MacKay-Marg tonometry. Gónioscopy revealed only mild trabecular pigmentation. The diffuse keratitis was felt to be most consistent with herpetic disciform disease, and the patient was started on a trial of systemic steroids. When seen 11 days later he was no better, and the steroids were discontinued.

The patient was seen next on 14 June 1977 with a dendritic epithelial defect. Treatment with vidarabine was begun again. A culture of the ulcer yielded HVH; indirect fluorescent antibody testing showed it to be type 2. On 1 September 1977 he developed a dendritic lesion of the inferotemporal cornea. This responded to treatment with vidarabine. In November 1977 the patient had a recurrence of the penile lesion, from which HVH-2 was cultured.

On 8 February 1978 a central epithelial defect was noted. Bacterial cultures showed no growth. Visual acuity was hand motions at 2 feet $(60 \mathrm{~cm})$. The ulcer, treated with topical polymyxin $B$, bacitracin, and neomycin (Neosporin) and subconjunctival gentamicin, healed over 10 days, but on 7 March 1978 the epithelium again broke down. This time the culture yielded pneumococci. In spite of subconjunctival and topical antibiotics the lesion failed to heal, and on 17 April 1978 a conjunctival flap operation was performed. The patient did well until September 1978, when the penile lesion recurred for the third time and persisted for 2 weeks. One week later he developed aching and redness in his left eye. The conjunctival flap prevented a view of the anterior chamber; the intraocular pressure was raised by tactile estimation. We felt that the patient had a reactivation of herpetic uveitis and treated him with cycloplegia. This produced symptomatic improvement. In November 1978 the patient's intraocular pressure was $32 \mathrm{mmHg}$ in the left eye, and he was given timolol.

\section{Discussion}

In 1975 Oh et al. reported the first 2 cases of cultureproved HVH-2 ocular infection in adults. ${ }^{5}$ Since that time 12 additional cases, ${ }^{1467}$ not including ours, have been described. In 3 of these ${ }^{14}$ no clinical data was included. The available information concerning the clinical course of 12 patients is summarised.

Three had blepharoconjunctivitis without corneal involvement. Of the 9 with corneal disease all had epithelial disease, and 6 had stromal pathology. Four of the 12 had uveitis.

Five of the 12 patients required a total of 8 surgical procedures. Two had conjunctival flaps: one for a persistent epithelial defect and the other for a corneal perforation. Of the 6 keratoplasties done 3 were for optical reasons, 2 were for structural purposes, and 1 was not specified.

Although a referral practice may tend to select for those patients with recalcitrant disease, this group of 12 patients with ocular HVH-2 appears to have had more severe and prolonged disease than the average patient with ocular HVH-1. There is experimental evidence to support this clinical observation. The conjunctivitis, corneal ulceration, pannus, and iritis that resulted from dropping HVH-2 on intact and abraded rabbit corneas were more marked and of longer duration than the changes produced by HVH-1. ${ }^{8}{ }^{9}$ Histological studies of these corneas showed stromal inflammatory infiltrates in type 2 infection to be deeper and more severe than in type 1 infection. Infiltration of the corneal endothelium, iris, and choroid by inflammatory cells was more frequent in eyes infected with type 2 than with type $1.0^{10}$

The spread of HVH-2 to different parts of the body may be via several routes: neural, haematogenous, and extrinsic. In our case, if the virus had been spread extrinsically from the penile lesion to the eye, one would have expected, as in other instances, ${ }^{6}$ blepharoconjunctivitis or keratitis to have been the first manifestation of the ocular infection. The fact that our patient's eye difficulties began with uveitis and that the corneal epithelial defect did not develop until several months later makes direct genito-ocular or genito-digital-ocular transmission unlikely, unless the initial corneal epithelial infection was subclinical.

Because of the distance between the penile lesion and the eye, neuronal spread is unlikely, though one cannot rule out neural transmission from the uvea to the cornea. Thus, haematogenous dissemination of the virus from the genital tract to the eye seems the most likely mode of transmission in our patient, though according to Nahmias and Roizman 
HVH-2 viraemia is an infrequent finding in a healthy adult. ${ }^{11}$ Eiferman et al. ${ }^{12}$ in a report of 2 cases of herpetic type 1 whitlow followed by dendritic keratitis, raised the possibility of haematogenous spread of virus from the fingers to the eye. They cited the work of Sery and Forster, ${ }^{13}$ who found that intravenous injection of HVH-1 in rabbits could cause dendritic keratitis about 10 days later. HVH-1 has been isolated from the blood buffy coat of 4 adults with vesicular stomatitis. ${ }^{14} \mathrm{HVH}-2$ has been detected in the blood of 2 adult patients with meningitis. ${ }^{15}$ Becker et al. studied the stages of haematogenous spread of HVH in children, though they do not specify whether the virus was type 1 or type 2.16 Blood-borne dissemination of HVH-2 may occur in newborn babies, ${ }^{17}$ and a case has been reported of fatal dissemination of HVH-2 infection in an apparently healthy 20-year-old woman who had necropsy evidence of thymic dysplasia. ${ }^{18}$ Our case suggests that haematogenous spread of HVH-2 may occur in healthy adults as well.

Supported in part by grant EY $02426-01$ and core grant EY 0179 from the National Institutes of Health.

\section{References}

1 Nahmias AJ, Visintine AM. Type 2 herpes simplex virus infections. Surv Ophthalmol 1976; 21 : 115-20.

2 Nahmias AJ, Dowdle WR. Antigenic and biologic differences in Herpesvirus hominis. Prog Med Virol 1968; 10: 110-59.

3 Dowdle WR, Nahmias AJ, Harwell RW, Pauls FP. Association of antigenic type of Herpesvirus hominis with site of viral recovery. $J$ Immunol 1967; 99: 974-80.

4 Hanna L, Ostler HB, Keshishyan H. Observed relationship between herpetic lesions and antigenic type of Herpesvirus hominis. Surv Ophthalmol 1976; 21 : 110-4.

5 Oh JO, Kimura SJ, Ostler HB. Acute ocular infection by type 2 herpes simplex virus in adults. Arch Ophthalmol 1975; 93: 1127-9.

6 Oh JO, Kimura SJ, Ostler HB, Dawson CR, and Smolin G. Oculogenital transmission of type 2 herpes simplex virus in adults. Surv Ophthalmol 1976; 21 : 106-9.

7 Neumann-Haefelin D, Sundmacher R, Wochnik, G, Bablok B. Herpes simplex virus types 1 and 2 in ocular disease. Arch Ophthalmol 1978; 96: 64-9.

8 Oh JO, Moschini GB, Okumoto M, Stevens T. Ocular pathogenicity of types 1 and 2 Herpesvirus hominis in rabbits. Infect Immun 1972; 5: 412-3.

9 Oh JO, Stevens TR. Comparison of types 1 and 2 Herpesvirus hominis infection of rabbit eyes: I. Clinical manifestations. Arch Ophthalmol 1973; 90: 473-6.

10 Stevens TR, Oh JO. Comparison of types 1 and 2 Herpesvirus hominis infection of rabbit eyes: II. Histopathologic and virologic studies. Arch Ophthalmol 1973; 90: 477-80.

11 Nahmias AJ, Roizman B. Infection with herpes-simplex viruses 1 and 2 . $N$ Engl $J$ Med 1973; 289: 667-74, 719-25, $781-9$.

12 Eiferman RA, Adams HG, Stover B, Wilkins T. Herpetic whitlow and keratitis. Arch Ophthalmol 1979; 97: 107981 .

13 Sery TW, Forster L. Viremia induction of herpes virus dendritic keratitis and uveitis. In Silverstein AM, O'Connor GR, eds. Immunology and Immunopathology of the Eye. New York: Masson, 1979.

14 Naraqi S, Jackson GG, Jonasson OH. Viremia with herpes simplex type 1 in adults. Ann Intern Med 1976; 85: $165-9$.

15 Craig CP, Nahmias AJ. Different patterns of neurologic involvement with herpes simplex virus types 1 and 2: Isolation of herpes simplex virus type 2 from the buffy coat of two adults with meningitis. J Infect Dis 1973; 127: 365-72.

16 Becker WB, Kipps A, McKenzie D. Disseminated herpes simplex virus infection. Am J Dis Child 1968; 115: $1-8$.

17 Nahmias AJ, Alford CA, Korones SB. Infection of the newborn with Herpesvirus hominis. Adv Pediatr 1970; 17: $185-226$.

18 Sutton AL, Smithwick EM, Seligman SJ, Kim D. Fatal disseminated Herpesvirus hominis type 2 infection in an adult with associated thymic dysplasia. Am J Med 1974; 56: $545-53$ 